\title{
MINIMAL COVERS AND ARITHMETICAL SETS ${ }^{1}$
}

CARL G. JOCKUSCH, JR. AND ROBERT I. SOARE

Abstract. If $\boldsymbol{a}$ and $\boldsymbol{b}$ are degrees of unsolvability, $\boldsymbol{a}$ is called $\mathbf{a}$ minimal cover of $\boldsymbol{b}$ if $\boldsymbol{b}<\boldsymbol{a}$ and no degree $c$ satisfies $\boldsymbol{b}<\boldsymbol{c}<\boldsymbol{a}$. The degree $a$ is called a minimal cover if it is a minimal cover of some degree $b$. We prove by a very simple argument that $0^{n}$ is not a minimal cover for any $n$. From this result and the axiom of Borel determinateness (BD) we show that the degrees of arithmetical sets (with their usual ordering) are not elementarily equivalent to all the degrees. We also point out how this latter result can be proved without $\mathrm{BD}$ when the jump operation is added to the structures involved.

Our notation is standard. We use $N$ to denote the set of all natural numbers and "l.u.b." to abbreviate "least upper bound."

To prove that $0^{n}$ is not a minimal cover for any $n$, it is convenient to prove a somewhat stronger result, both for the sake of extra corollaries and in order to have a sufficiently strong inductive hypothesis in the proof.

TheOREM 1. If $a \geqq 0^{n}$ and $a$ is a minimal cover of $b$, then $b \geqq 0^{n}$.

Proof. The proof is by induction on $n$. The theorem is trivial for $n=0$. We now assume the theorem for $n=k$ and prove it for $n=k+1$. Suppose $a \geqq 0^{k+1}$ and $\boldsymbol{a}$ is a mini nal cover of $b$. Let $c=1$.u.b. $\left\{0^{k+1}, b\right\}$. Clearly $b \leqq c \leqq a$ and so $c=b$ or $c=a$. If $c=b$, then $0^{k+1} \leqq b$ as required. Now suppose $c=a$. We claim that then $a$ is r.e. in $b$. Since $a \geqq 0^{k}$, it follows from the induction assumption that $b \geqq 0^{k}$. Thus $0^{k+1}$ is r.e. in $b$, since it is r.e. in $0^{k}$. Hence $a$ is the l.u.b. of two degrees r.e. in $b$ and so must be r.e. in $b$. By relativizing the theorem of Friedberg and Muchnik that no r.e. degree is minimal [6, p. 66, Corollary 1], it now follows that $a$ is not a minimal cover of $b$, contrary to hypothesis.

COROLlaRy 1. If $\mathbf{a} \geqq 0^{n}$ and $a$ is r.e. in $0^{n}$, then a is not a minimal cover.

Received by the editors October 21, 1969.

AMS Subject Classifications. Primary 0270, 0277.

Key Words and Phrases. Recursive function, degree of unsolvability, arithmetical hierarchy, axiom of determinateness.

1 This research was supported in part by National Science Foundation grants GP-7421 and GP-8866. The authors wish to thank D. A. Martin, A. H. Lachlan, and the referee for helpful suggestions and information. 
Proof. Assume $a \geqq 0^{n}, a$ is r.e. in $0^{n}$, and $a$ is a minimal cover of $b$. Then $b \geqq 0^{n}$ by the theorem, so $a$ is r.e. in $b$. As before, this is impossible.

Corollary 2. If a is a minimal upper bound to $\left\{0^{n}: n \in N\right\}$, then $a$ is not a minimal cover.

Proof. Immediate from the theorem.

Corollary 3. There exist $2^{N_{0}}$ degrees which are not minimal covers.

Proof. This follows from Corollary 2 because Sacks has shown $[6$, p. 131] that every countable ascending sequence of degrees has $2^{\aleph_{0}}$ minimal upper bounds.

Let $a$ be the collection of all degrees of arithmetical sets and let $D$ be the collection of all degrees. In what follows we use the symbol $\leqq$ both for the ordering of all degrees and for the restriction of this ordering to $a$.

The axiom of Borel determinateness (BD) states that the game $G_{2}(\mathcal{K})$ (as defined in [5, p. 206]) is determined for every Borel subset $\mathfrak{K}$ of $2^{\omega}$. D. A. Martin has shown [4] that BD is implied by the existence of measurable cardinals and in fact by much weaker "large cardinal" assumptions. Thus the following corollary is also a consequence of these assumptions.

Corollary 4. (Assuming BD.) The structure $\langle Q, \leqq\rangle$ is not elementarily equivalent to $\langle D, \leqq\rangle$.

Proof. We shall need the following result of Martin [3, p. 688]. Let $\mathscr{T}$ be a set of degrees whose union is a Borel subset of $2^{\omega}$, and suppose $\mathfrak{T}$ satisfies:

$$
(\forall a)(\exists b)[b \geqq a \wedge b \in \mathscr{T}] .
$$

Then if BD holds, $\mathfrak{T}$ must satisfy the stronger statement:

$$
(\exists a)(\forall b)[b \geqq a \rightarrow b \in \mathfrak{N}] .
$$

In particular now let $\mathfrak{M}$ be the collection of all degrees which are minimal covers. The union of $\pi$ is easily shown to be Borel and in fact $\Sigma_{5}^{0}$ in the arithmetical hierarchy. Also (1) is true by the relativized version of Spector's minimal degree construction [7, Theorem 4]. Thus, if $\varphi$ is the first order statement which asserts (2), $\varphi$ holds in the structure $\langle\mathscr{D}, \leqq\rangle$. On the other hand, $\varphi$ does not hold in $\langle a, \leqq\rangle$ because no $0^{n}$ is a minimal cover.

It follows from Corollary 4 that $\langle Q, \leqq\rangle$ is not an elementary substructure of $\langle\mathcal{D}, \leqq\rangle$. This answers, modulo $\mathrm{BD}$, a question 
raised by G. E. Sacks. We do not know whether Corollary 4 is provable without $\mathrm{BD}$, but it is easy to give an absolute proof of the following weaker form of Corollary 4.

PROPOSITION 1. The structure $\left\langle D, \leqq,^{\prime}\right\rangle$ is not elementarily equivatent to $\langle Q, \leqq, '\rangle$.

Proof. Let $\varphi$ be the sentence:

$$
(\exists a)(\exists b)(\forall c)\left[c \leqq a \wedge c \leqq b \rightarrow c^{\prime} \leqq a \wedge c^{\prime} \leqq b\right] .
$$

Obviously $\varphi$ does not hold in $\left\langle Q, \leqq,^{\prime}\right\rangle$. On the other hand, by the proof of a theorem of Kleene and Post [2, Theorem 3], the set of degrees $\left\{0^{n}: n \in N\right\}$ has a pair of upper bounds $a, b$ such that every lower bound to $\{a, b\}$ is in fact $\leqq 0^{n}$ for some $n$. Hence $\varphi$ holds in $\langle D, \leqq, '\rangle$.

In contrast to the foregoing, the structures $\left\langle Q,{ }^{\prime}\right\rangle$ and $\left\langle D,{ }^{\prime}\right\rangle$ are elementarily equivalent. This can be shown by an elimination of quantifiers argument using a slight extension of Friedberg's completeness criterion [1]. The proof also shows that the common theory of these two structures is decidable.

In closing we consider possible generalizations and analogues for Theorem 1. We would like to extend Theorem 1 from the arithmetical hierarchy to the hyperarithmetical hierarchy. However, we are unable to decide even whether $0^{\omega}$ is a minimal cover. The root of the difficulty here is that $\left\{0^{n}: n \in N\right\}$ has no l.u.b.

On the other hand, some ascending sequences of hyperdegrees do have l.u.b.'s, and thus it is easy to extend Theorem 1 into the transfinite for hyperdegrees. To this end we define hyperdegrees $h_{\alpha}$ for certain countable ordinals $\alpha$ by transfinite induction. Let $h_{0}$ be the minimum hyperdegree, and let $h_{\alpha+1}$ be the hyperjump of $h_{\alpha}$, provided $h_{\alpha}$ is defined. Finally, if $\lambda$ is a limit ordinal, let $h_{\lambda}$ $=1 . u . b .\left\{h_{\alpha}: \alpha<\lambda\right\}$ provided all $h_{\alpha}, \alpha<\lambda$, are defined and the l.u.b. exists. (It is known that $h_{\alpha}$ exists exactly for $\alpha<\omega_{1}^{E_{1}}$.) Now if the terminology for Theorem 1 is modified in the obvious way for hyperdegrees, then it still holds when $a, b$ are hyperdegrees and $0^{n}$ is replaced by $\boldsymbol{h}_{\alpha}$ for any $\alpha$ such that $\boldsymbol{h}_{\alpha}$ is defined. The argument is trivial at limit ordinals and virtually the same as before (with " $\Pi_{1}^{1}$ " replacing "r.e.") at successor ordinals.

\section{REFERENCES}

1. R. M. Friedberg, A criterion for completeness of degrees of unsolvability, J. Symbolic Logic 22 (1957), 159-160. MR 20 \#4488. 
2. S. C. Kleene and E. L. Post, The upper semi-lattice of degrees of recursive unsolvability, Ann. of Math. (2) 59 (1954), 379-407. MR 15, 772.

3. D. A. Martin, The axiom of determinateness and reduction principles in the analytical hierarchy, Bull. Amer. Math. Soc. 74 (1968), 687-689. MR 37 \#2607. 291.

4. - Measurable cardinals and analytic games, Fund. Math. 66 (1970), 287-

5. J. Mycielski, On the axiom of determinateness, Fund. Math. 53 (1963/64), 205224. MR $28 \# 4991$.

6. G. E. Sacks, Degrees of unsolvability, Ann. of Math. Studies, no. 55, Princeton Univ. Press, Princeton, N. J., 1963.

7. C. Spector, On degrees of recursive unsolvability, Ann. of Math. (2) 64 (1956), 581-592. MR 18, 552; MR 18, 1118.

UNIVERSITY OF ILLINOIS, URBANA, IlLINOIS 61801

University of Illinois at Chicago Circle, Chicago, Illinois 60680 